\section{THE MANAGEMENT OF ABDOMINAL OPERATIONS IN INFANTS.}

\section{By RODNEY MAINGOT, F.R.c.s.}

Surgeon, Royal Waterloo Hospital.

THIS is a very large subject, comprising, as it does, the pre- and post-operative treatment and special measures which may be required for individual cases. It is quite impossible in the time at my disposal to enlarge fully upon all the details embraced by the title. So I propose to discuss some of the routine and special measures usually advocated before and after operations on infants; also to give a brief synopsis or scheme which may prove helpful in the management of cases of congenital hypertrophic stenosis of the pylorus and general peritonitis.

There is no reason why the management of these cases should in any way differ from that of adults. It is largely a question of minor details, and it is my intention to indicate these as I deal with the particular subjects.

The following is a brief scheme of preoperative treatment which will have to be varied to meet the special requirements of special cases. No rule of thumb method, or hard and fast rules, are encouraged in the management of any one case; variations, modifications and adaptations are necessary if the best results are to be achieved. In the pre-operative management, too, some distinction will have to be made between chronic and acute abdominal cases.

\section{SCHEME FOR PRE-OPERATIVE MANAGEMENT.}

(I) The usual methods adopted for preventing operative shock:-

(a) Avoid starvation and drastic preoperative purgation. Food is, as a rule, allowed up to two or three hours before the anæsthetic is given, and should be of such a nature as to leave little or no residue in the stomach. Sugars, therefore, will be given in quantity, and will form the basis of most of the feeds. Water is given generously up to two hours before the operation.

(b) The liberal administration of fluids and alkalies before operation is imperative.

(c) Gas and oxygen and ether, or ether, are the anæsthetics of choice, and do not produce so much shock and metabolic disturbances as chloroform. Spinal anæsthetics in children are unsatisfactory, and local anæsthetics, owing to obvious reasons, are rarely, if ever, employed.

(d) Unnecessary loss of blood is to be carefully avoided during operation.

(e) All abdominal operations in children should be conducted as rapidly as possible, compatible with thoroughness.

$(f)$ All unnecessary or rough manipulations, either of the abdominal wall, mesentery, or intestines, should be sedulously guarded against.

(g) Measures should be adopted to conserve the body heat during the operation.

(h) If shock is present during an operation, salines should be given subcutaneously or intravenously.

(2) Measures for Preventing Acidosis.The urine should be carefully examinèd before every operation on infants, and if any trace of acetone is present, or if acidosis is to be feared as a result of the operation, then the administration of the following is called for.

(a) Glucose by the mouth, rectum, or subcutaneously. Five per cent. glucose in water is given frequently by the mouth. Two per cent. glucose in $8 \mathrm{oz}$. of saline is given every four or five hours while the child is awaiting operation.

(b) Either 30 gr. of sodium bicarbonate or of alkaline sodium phosphate every four hours by the mouth if the vomiting is severe, or 2 drachms of one of the above in $8 \mathrm{oz}$. of water may be injected into the rectum at six-hourly intervals. Sodium bicarbonate or alkaline sodium phosphate are not injected 
subcutaneously, as they very often produce severe pain, and in some instances cellulitis or actual gangrene of the skin. Rectal infusions are, as a rule, only practicable in children over 6 years of age. In younger children the fluid is hardly ever retained, and it is waste of time to persevere with these, as coughing, straining, or crying will result in the infusions being passed.

(c) Gastric lavage.

(3) Hypodermic Injection before Operation.-Morphia is a very dangerous drug for children, and is practically never given before operation. Atropine, however, is given as a routine measure, half-an-hour before operation, in the following doses :-

During the first year of life $\ldots \frac{1}{20} \mathrm{gr}$.

" " second year of life... $1_{1 \frac{1}{5} \delta} \mathrm{gr}$.

After the ," , , ,.. $\frac{1}{10} \mathrm{gr}$.

Atropine is a mild cardiac stimulant, and is a most effective drug in drying up bronchial and salivary secretions, and also facilitates the administration of inhalation anæsthetics.

(4) Management of Bowels. - The bowel is emptied by means of an enema twelve hours before the operation. Purgatives by the mouth are rarely, if ever, necessary, except perhaps in the most exceptional cases, when a full dose of castor oil may be used. The best enema is one containing $2 \mathrm{oz}$. of magnesium sulphate and $4 \mathrm{oz}$. of glycerine, in one quart of warm water. In cases of peritonitis or obstruction, purges by the mouth are to be deprecated, and although one or two enemata may be called for to clear out the lower gut, their repetition is not recommended, as they do more harm than good.

(5) Pre-operative Rest.-Pre-operative rest and sleep are of incalculable value, and such drugs as syrup of chloral hydrate, chloretone, or small doses of nembutal are sometimes given. If, however, the patient is vomiting, the following rectal sedatives may be tried : pot. brom. 2 gr. and aspirin $2 \mathrm{gr}$. in $6 \mathrm{oz}$. of warm water, this quantity being given to a child aged 2, and the bromide and aspirin increased by 2 gr. for every year of the child's age.

(6) Feeding before Operation.-As previously stated, food is allowed up to two or three hours before operation, but in certain cases, e.g., acute peritonitis, food should not be given. The feeds are given frequently, and the bulk should consist of easily assimilable sugars, as glucose, dextrose, \&c. Fluids are allowed up to an hour or two before the child is taken to the operating theatre Starvation is now never enjoined, as it is a potent factor in producing acidosis.

\section{SCHEME For POST-Operative MANAGEMENT.}

The post-operative management of a case will depend largely on the nature of the operation that has been performed, and upon the disease from which the child is suffering, but the following points demand special consideration.

(I) Vomiting.-After the operation I drm. of glucose water, to which has been added $5 \mathrm{gr}$. of sodium bicarbonate, should be given every five or ten minutes for two hours, and after this a tumblerful of warm sodium bicarbonate solution to produce vomiting. If, however, vomiting is persistent or intractable, the stomach may require washing out through a Ryle's stomach tube. The usual methods of treating acidosis should be employed to counteract the vomiting, which, if unrelieved by these measures, will suggest the presence of some grave intra-abdominal lesion, possibly necessitating some further operative or special procedure.

(2) Pain.-Syrup of chloral hydrate is probably the best sedative, and can be prescribed in doses up to I drm. for small infants. Sedatives introduced into the rectum are seldom retained, and this method should not be employed as a routine, although in older children the aspirn and bromide mixture mentioned above is very efficacious.

(3) Bowels after Operation.-Twelve hoúrs 
after the operation, a flatus tube should be passed, and after this at intervals of every three or four hours. After twenty-four hours, if there is no vomiting, paraffin in small doses is given two or three times a day, and is continued for the next week or so. After forty-eight hours an enema is administered; if there is no result from the enema another one may be tried, or an ox-gall enema substituted in a few hours' time. Enemata should be persisted in, and given every morning until the bowels are working naturally, or aided by some such mild purgative as syrup of figs, senna, or cascara evacuant.

(4) Wound.-The wound should be inspected at the end of twenty-four hours. If all is well, it remains undisturbed until the fourth or fifth day. Metal clips are removed on the fourth or fifth day; interrupted stitches on the seventh or eighth day; and subcuticular or deep tension stitches on the twelfth day. Strict aseptic and antiseptic precautions are observed on each occasion that the wound is dressed. When all the clips or stitches have been withdrawn, the wound and the area around are thickly smeared with double strength calamine lotion, and a square of lint or gauze, well soaked in the solution, is applied once or twice every day until the wound is firmly healed. An abdominal dressing or support may be required for a week or two after the patient leaves hospital. In certain cases, however, where some special abdominal dressing or paint has been employed directly to the wound, it should be left undisturbed for four or five days.

(5) Radiant Heat.-During the first three or four days following operation, radiant heat applications to the abdomen for ten to twenty minutes three times a day are recommended, and this should be a routine procedure. It diminishes shock, aids in the expulsion of flatus, and adds to the comfort of the patient.

(6) Tonic Treatment.-It is always wise to give the patient an alkaline or acid tonic during the period of convalescence, and in certain cases intramuscular injections of iron and arsenic are prescribed.

\section{Pyloric STENOSIS IN INFANTS.}

The æetiology of this condition at the present time is not fully understood, although it is assumed that the abnormality of the pylorus is a congenital one. The pylorus is thickened, due to hypertrophy of the sphincter muscle. The stomach is enlarged and gastritis is usually present. Eighty per cent. of cases occur in males, and the disease is more frequent in first-born children.

\section{Signs and Symptoms.}

( I) Wasting.--Signs and symptoms usually come on from one to six weeks after birth, but may be delayed until the sixteenth week or later. The child rapidly loses weight, the skin becomes inelastic and drawn into folds, giving rise to a wizened appearance.

(2) Vomiting.-The vomiting is projectile, and large quantities of fluid are ejected at each vomit.

(3) Constipation.-This is generally a marked feature.

(4) Visible Peristalsis.-The waves pass from left to right.

(5) Pyloric Tumour.-This is the main diagnostic sign.

(6) Tetany.

\section{Treatment.}

These cases are no longer to be regarded as surgical emergencies, but a deliberate plan of treatment, carefully and methodically worked out, should be undertaken in order to improve the patient's general condition, and make him fit to withstand the operation with every hope of success. It is surprising how these children will rapidly improve and gain weight under a systematic course of treatment lasting some three or four days. The pre-operative treatment may be briefly outlined as follows :-

(I) Stomach to be washed out two or three times a day through a Ryle's stomach tube.

(2) Only small frequent sips of sugar water or diluted milk to be given by-mouth. 
(3) The generous administration of rectal and subcutaneous salines.

(4) Atropine, $\frac{1}{1} \frac{1}{0} \mathrm{gr}$., or belladonna, Iom by the mouth, two or three times a day.

(5) In desperate cases, blood transfusion. Rammstedt's operation is the one of choice and offers the most speedy and certain cure for the disease. There can be little doubt that the best results are obtained when the operation is performed at the earliest possible moment after the onset of the symptoms, and after a preliminary intensive course of medical treatment. The following are the methods of feeding recommended by Poynton, Higgins and Brydson (Lancet, I924, ccvi, 215), and adopted after operation.

Breast Feeding.-Operation, ro.30 a.m.; return to ward, II a.m.; milk drawn from mother four hours later.

\begin{tabular}{|c|c|}
\hline First Day. & $\begin{array}{l}3 \text { to } 8 \text { p.m. } \\
9 \text { to Io p.m. } \\
\text { I I and } \text { I } 2 \text { p.m. }\end{array}$ \\
\hline Second Day. & $\begin{array}{l}\mathrm{I} \text { and } 2 \text { a.m. } \\
3 \text { and } 5 \text { a.m. } \\
7 \text { and } 9 \text { a.m. }\end{array}$ \\
\hline
\end{tabular}

To breast for three minutes, time varies

$$
5 \text { p.m. ... } \ldots \quad \xi \text { iss }
$$

To breast for five to seven minutes

$$
7.30 \mathrm{p} . \mathrm{m} . \quad \ldots \quad \xi \text { iss }
$$

Continue two and a half-hourly for twelve hours

Third Day. 7.30 a.m. (three-hourly for twelve hours)

Breast five to seven minutes

$$
\text { 7,30 p.m. (three-hourly) }
$$

Breast for quarter hour or twenty minutes

These babies are weighed before and after feeds for first few days.

\section{Bottle-Feeding. A Reliable Skimmed Dried Milk.}

Operation I0.30 a.m., return to ward i a.m.; four hours after commence feeding.

$$
\begin{array}{cccc}
\text { First Day. } \quad 3 \text { to } 8 \text { p.m. } & \ldots & 3 \mathrm{i} \text { (hourly) } \\
9 \text { and Io p.m. } & \ldots & 3 \text { ii } \\
\text { I I p.m. to r a.m.... } & 3 \text { iii (hourly) }
\end{array}
$$

Second Day. $\quad 2$ a.m. ... $\quad \ldots \quad 3$ iii

3 and 5 a.m. $\ldots 3$ iv

7 and 9 a.m. $\quad \ldots \quad 3$ vi

I I a.m., I p.m.

and 3 p.m. ... $\xi \mathrm{i}$

5 and 7.30 p.m... $\xi i, \xi$ ii

Continue two and a half-hourly for twelve hours

Third Day. Dried milk (half

$$
\text { cream) } \ldots 3 \text { i to } \xi \mathrm{i}
$$

7.30 a.m. ... $\xi$ iss (three-hourly for 12 hours)

7.30 p.m. ... 3 ii three-hourly

Increase to $\xi$ iiss or $\xi$ iii according to age. General Peritonitis.

The commonest cause of acute suppurative peritonitis in infants is acute appendicitis. This disease has a higher mortality than in adults. Special points to be noted in the management :-

(I) If vomiting is severe, the stomach contents should be thoroughly aspirated, and a stomach tube left in situ.

(2) Nopurgatives by the mouth or enemata to be given before operation.

(3) As much subcutaneous saline as possible should be injected.

(4) Large doses of anti-gas gangrene serum (B. welchii) are given. The usual dose in a severe case is 40 c.c. intramuscularly before operation. Further doses of 40 c.c. are given intramuscularly daily after operation until the bowels are working naturally, or until all signs of toxæmia are at an end.

(5) Drainage Tube.-If a drainage tube has been inserted into the abdominal cavity in an infant, it is unwise to remove it and replace it by a smaller one. This procedure is exceedingly painful and causes a great deal of shock. It is far better to rotate the tube at the end of twenty-four hours and shorten it daily.

(6) Wound.-The wound and the abdominal wall should be thickly coated with zinc and castor-oil mixture (equal parts) to protect the skin from discharges from the tube and to prevent blistering from the 
frequent applications of hot fomentations and radiant heat.

(7) Bowels.-Purgatives by the mouth are to be avoided, and reliance should be placed on enemata to achieve an action of the bowels.

\section{POST-GRADUATE NEWS.}

THE following Special Courses will be given during October :-

Central London Throat, Nose and Ear Hospital, Gray's Inn Road, W.C.I.

A Course in Diseases of the Ear, Nose and Throat will be given at the above hospital from October 5 to October $3 \mathrm{I}$. The Clinical Course will include, during the first week only, Lectures in Anatomy and Physiology, and a Course in Methods of Examination and Diagnosis. The fee for the first week only is $£^{2} 2 \mathrm{~s}$., and for the four weeks $£ 55$ s., including all Lectures and attendance at Clinics and Operations. In addition, the following special classes will be given: Operative Surgery, fee $£_{7} 7 \mathrm{~s}$. (strictly limited); Pathology, fee $£ 5$ 5s. (strictly limited); and Peroral Endoscopy, fee $£ 66$ s. (strictly limited).

Hospital for. Tropical Diseases, 25, Gordon Street, W.C.I.

An all-day Course in Tropical Diseases will be held from October 5 to October 24, including Lectures, Museum Demonstrations, and the study of Clinical Cases. Fee $£ 88 \mathrm{~s}$.

St. John's Hospital, Leicester Square, W.C.2.

A Course in Dermatology will be given from October 12 to November 7, consisting of attendance at the clinics every afternoon at 2 p.m. and 6 p.m., and Lectures on Tuesdays and Thursdays at 5 p.m. Fee $f_{I}$ Is. For those interested in Pathology, special demonstrations will be arranged at a fee of $E_{4} 4$ s.
Chelsea Hospital for Women, Arthur Street, S.W.3.

A Course in Gynæcology will be held from October 12 to October 24. Most mornings and afternoons are occupied with Lectures and Demonstrations in the Operating Theatre. Fee $£ 55 \mathrm{~s}$.

National Hospital for Diseases of the Heart, Westmoreland Street, W.

A fortnight's Course in Diseases of the Heart will be held from October 12 to October 24, occupying the whole of each day. Fee $£ 7$ \%s. The Course is strictly limited to twenty.

Hospital for Sick Children, Great Ormond Street, W.C.I.

A morning Course in Diseases of Children will be held from October I9 to October $3 \mathrm{I}$, including Lectures and Demonstrations. Fee $£_{5} 5 \mathrm{~s}$. As this Course will only be held if a minimum of ten post-graduates enrol, candidates are particularly requested to send in their names in plenty of time to avoid cancellation of the Course.

Prince of Wales's Hospital, Tottenham, N.I5.

A "refresher" Course in Medicine and Surgery will be held from October I 2 to 24, occupying the whole of each day, from ro.30 a.m. to 5.30 p.m., with special Demonstrations, Clinical Practice, and Formal Lectures. Though this Course is considered especially suitable for general practitioners, it is not restricted in any way and is open to men and women. Fee $£ 55$ s., or $€ 33 \mathrm{~s}$. for either week.

\section{M.R.C.P. Evening Course.}

A Course of eighteen Lectures especially suitable for M.R.C.P. candidates will be given on Monday and Wednesday evenings at 8.30 (unless otherwise indicated), at the Medical Society of London, II, Chandos 